\title{
Design and characterization of a
}

recirculating liquid-microjet photoelectron spectrometer for multiphoton ultraviolet photoelectron spectroscopy

Cite as: Rev. Sci. Instrum. 90, 083104 (2019); https://doi.org/10.1063/1.5099040

Submitted: 05 April 2019 . Accepted: 20 July 2019 . Published Online: 09 August 2019

Jamie W. Riley ${ }^{(D)}$, Bingxing Wang (D), Michael A. Parkes (D), and Helen H. Fielding (D)

\section{ARTICLES YOU MAY BE INTERESTED IN}

Cavity-enhanced high harmonic generation for extreme ultraviolet time- and angleresolved photoemission spectroscopy

Review of Scientific Instruments 90, 083001 (2019); https://doi.org/10.1063/1.5090507

Quantification of atomic hydrogen anion density in a permanent magnet based helicon ion source (HELEN) by using pulsed ring down spectroscopy

Review of Scientific Instruments 90, 083103 (2019); https://doi.org/10.1063/1.5093914

Advances in liquid phase soft-x-ray photoemission spectroscopy: A new experimental setup at BESSY II

Review of Scientific Instruments 88, 073107 (2017); https://doi.org/10.1063/1.4990797

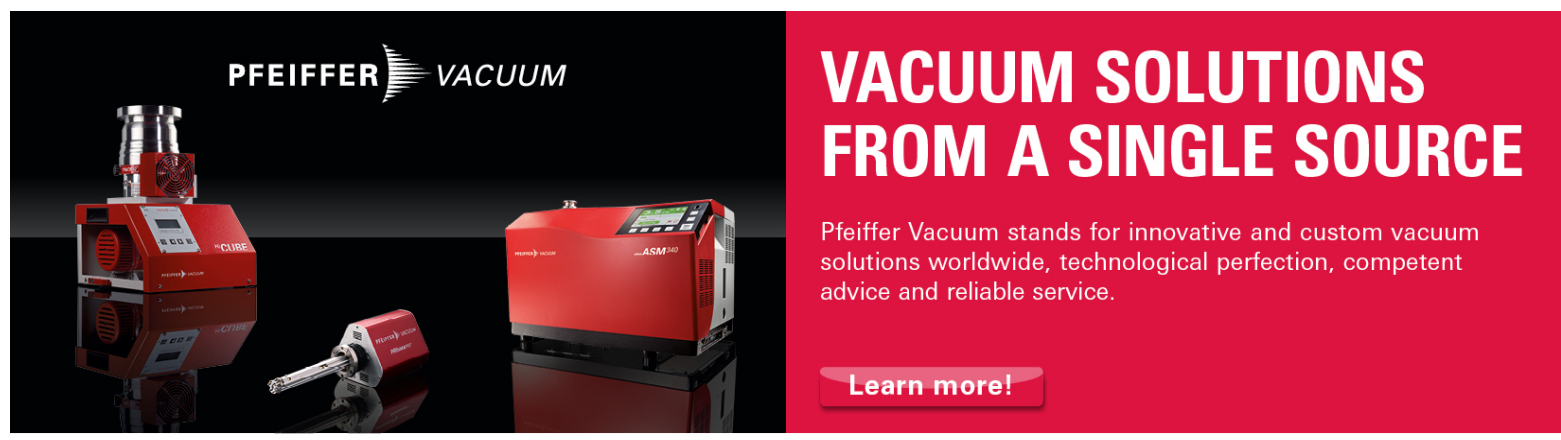




\title{
Design and characterization of a recirculating liquid-microjet photoelectron spectrometer for multiphoton ultraviolet photoelectron spectroscopy
}

\author{
Cite as: Rev. Sci. Instrum. 90, 083104 (2019); doi: 10.1063/1.5099040 \\ Submitted: 5 April 2019 - Accepted: 20 July 2019 • \\ Published Online: 9 August 2019
}

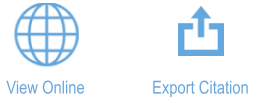

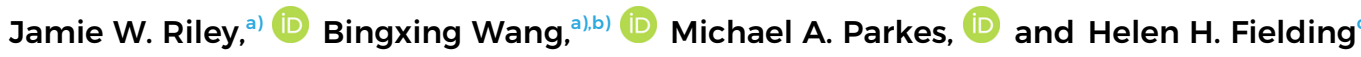

\section{AFFILIATIONS}

Department of Chemistry, University College London, 20 Gordon Street, London, WClH OAJ, United Kingdom

a) Contributions: J. W. Riley and B. Wang contributed equally to this work.

b) Current address: College of Chemistry and Chemical Engineering, Henan Institute of Science and Technology, Eastern Hualan

Avenue, Xinxiang, 453003, China.

${ }^{c)}$ Electronic mail: h.h.fielding@ucl.ac.uk

\begin{abstract}
A new recirculating liquid-microjet photoelectron spectrometer for multiphoton ultraviolet photoelectron spectroscopy is described. A recirculating system is essential for studying samples that are only available in relatively small quantities. The reduction in background pressure when using the recirculating system compared to a liquid-nitrogen cold-trap results in a significant improvement in the quality of the photoelectron spectra. Moreover, the recirculating system results in a negligible streaming potential. The instrument design, operation, and characterization are described in detail, and its performance is illustrated by comparing a photoelectron spectrum of aqueous phenol recorded using the recirculating system with one recorded using a liquid nitrogen cold-trap.
\end{abstract}

Published under license by AIP Publishing. https://doi.org/10.1063/1.5099040

\section{INTRODUCTION}

There is considerable interest in improving our understanding of how molecules respond to ultraviolet (UV) light, both from a fundamental point of view and as a result of relevance in nature and technology; important examples include photosynthesis, photovoltaics, vision, and imaging. A great deal of our detailed understanding of the UV photoresponse of molecules has come from gas-phase experiments and calculations involving isolated molecules, free from complex interactions with solvent or protein environments. However, electronically excited states can be exquisitely sensitive to their environment, particularly polar solvents such as water, the most important medium in chemistry and biology. The extent to which dynamical insights obtained from detailed gas-phase studies can be used to inform our understanding of the dynamics of photoexcited molecules in chemically and biologically relevant environments is a subject of much current discussion.
Understanding the UV photoresponse of a molecule requires a detailed understanding of its electronic structure and electronic relaxation pathways. Experimentally, a direct way of determining the electronic structure is through the measurement of electron binding energies using photoelectron spectroscopy. Femtosecond time-resolved photoelectron spectroscopy has proved to be a particularly valuable tool for tracking electronic relaxation pathways in gas-phase molecules ${ }^{2-6}$ and molecules on surfaces. ${ }^{7,8}$ For a long time, photoelectron spectroscopy was limited to low vapor pressure samples. However, the introduction of liquid-microjets in the late 1990s made it possible to extend photoelectron spectroscopy techniques to probe the electronic structure of molecules in solution. ${ }^{9}$ Liquidmicrojet x-ray photoelectron spectroscopy has been used widely to probe the electronic structure of liquid water, salt solutions, and some organic molecules and nanoparticles. ${ }^{10,11}$ A handful of groups are using liquid-microjet UV photoelectron spectroscopy to probe the electronic structure and relaxation dynamics of UV photoexcited molecules in solution. ${ }^{12-34}$ This approach has the added 
advantage that resonance-enhanced multiphoton ionization of a molecule enhances the signal-to-noise ratio of the photoelectron spectrum of the molecule and is essentially free from the solvent background.

Most liquid-microjet photoelectron spectroscopy experiments involve running the liquid through a thin fused silica liquid-microjet nozzle into vacuum and then freezing it on a cold-trap filled with liquid nitrogen. However, for samples that are only available in small quantities, such as proteins, nanoparticles, and bespoke molecules, it becomes desirable to recirculate the liquid rather than freeze it out. This paper describes the design and construction of a new apparatus combining a recirculating liquid-microjet and a magnetic bottle photoelectron spectrometer for multiphoton UV photoelectron spectroscopy. The design and technical details are described in Sec. II. Section III explains the operation, calibration, and characterization of the instrument, including a comparison between measurements of the streaming potential using the recirculating system and a liquid nitrogen cold-trap (Sec. III D). In Sec. IV, we illustrate the performance of the instrument by comparing photoelectron spectroscopy measurements of aqueous phenol made using the recirculating system and a liquid nitrogen cold-trap.

\section{INSTRUMENT DESIGN AND TECHNICAL DETAILS}

A cross section of the liquid-microjet photoelectron spectrometer is presented in Fig. 1. The main components are (i) the laser-liquid interaction chamber, (ii) the photoelectron time-offlight chamber, and (iii) the photoelectron detection chamber. The interaction, time-of-flight and detection chambers are constructed from 316 stainless steel and have volumes of around $1.6 \times 10^{-2} \mathrm{~m}^{3}$, $1.5 \times 10^{-2} \mathrm{~m}^{3}$ and $3.1 \times 10^{-3} \mathrm{~m}^{3}$, respectively. The chambers are differentially pumped to maintain pressures of around $1 \times 10^{-6} \mathrm{mbar}$, $2 \times 10^{-8}$ mbar and $3 \times 10^{-8}$ mbar, respectively. During operation of the liquid-microjet with the recirculating system (Sec. II A), these pressures increase to $2 \times 10^{-5} \mathrm{mbar}, 5 \times 10^{-7} \mathrm{mbar}$, and $6 \times 10^{-7}$ mbar. If the liquid-microjet is operated with a liquid nitrogen cold-trap to freeze out the liquid, the pressure in the interaction chamber rises to a higher pressure of $1-2 \times 10^{-4}$ mbar. This has a significant impact on the performance of the liquid-microjet photoelectron spectrometer (Sec. IV).

\section{A. Liquid-microjet}

The liquid-microjet assembly was purchased from Microliquids $\mathrm{GmbH}$. The liquid is pumped through $1 / 16^{\prime \prime}$ polyether ether ketone (PEEK) tubing with an approximate length of $1 \mathrm{~m}$ by a highperformance liquid chromatography (HPLC) pump and injected into the laser-liquid interaction region through a $25 \mathrm{~mm}$ long quartz nozzle with internal diameter of $10-50 \mu \mathrm{m}$. The internal diameter of the nozzle is selected to minimize evaporation and is therefore dependent on the vapor pressure of the liquid. ${ }^{36}$ The HPLC pump has a built-in pulsation damper to remove any bubbles that form inside the tubing; it works efficiently at pressures above 70 bar.

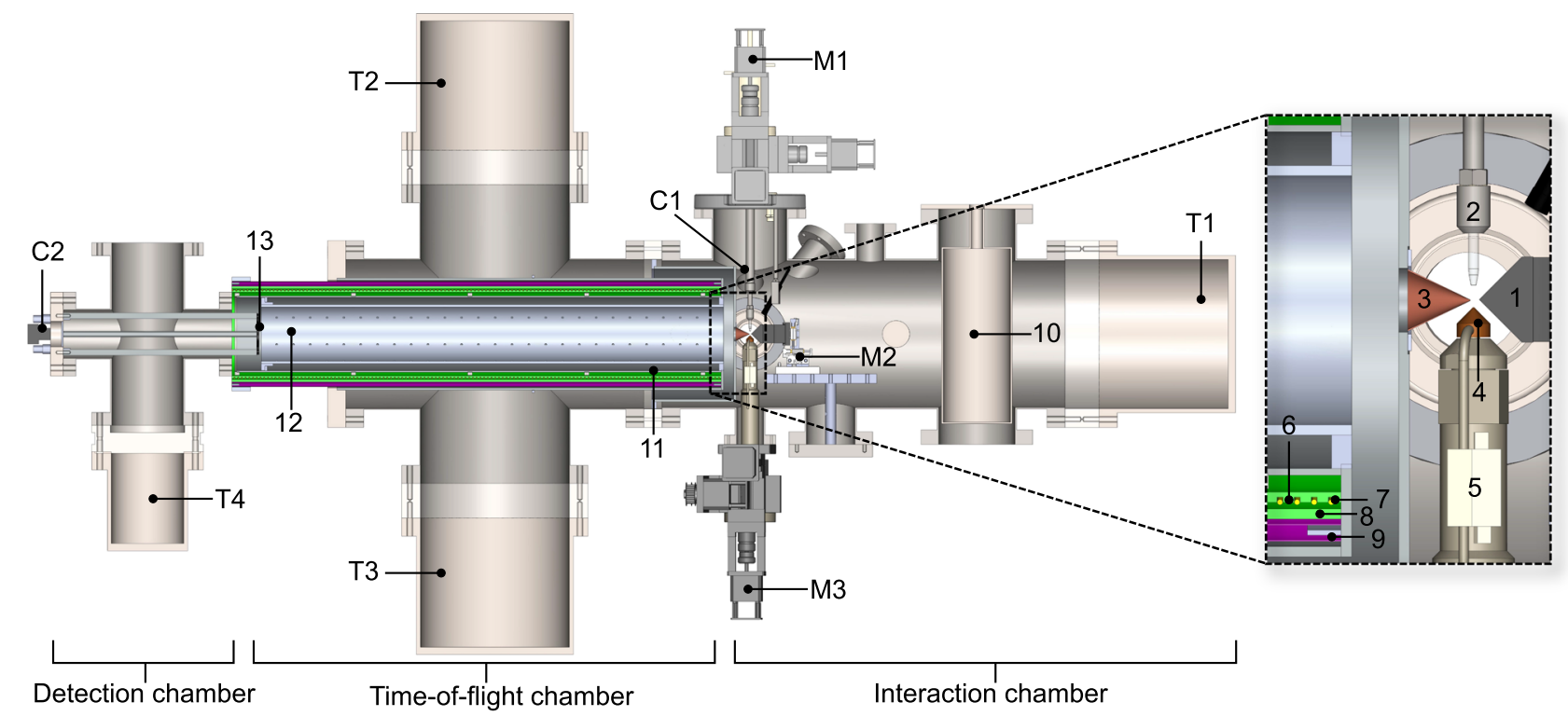

FIG. 1. Cross section of the liquid-microjet photoelectron spectrometer that combines a liquid-microjet assembly (Microliquids GmbH) and a magnetic-bottle photoelectron spectrometer. The magnified inset highlights the following components: (1) magnet, (2) nozzle, (3) skimmer, (4) catcher tip, (5) heater, (6) inner PTFE sleeve with a groove for solenoid, (7) solenoid, (8) outer PTFE tube, and (9) double $\mu$-metal tube. The interaction chamber has three separate xyz-manipulators for alignment: (M1) for the liquid-microjet, (M2) for the magnet, and (M3) for the catcher assembly. To monitor the alignment, a CMOS camera (C1) is located on the far side of the instrument. Using a $1000 \mathrm{I} \mathrm{s}^{-1}$ turbomolecular pump (T1) and cryogenic pumping with a cold trap (10), a pressure of around $2 \times 10^{-5}$ mbar is maintained in the interaction chamber when the liquid-microjet is running (see text). The time-of-flight chamber consists of two concentric tubes: a flight tube (11) and a drift tube (12). The high-vacuum conditions of the drift tube are maintained using two $600 \mathrm{I} \mathrm{s}^{-1}$ turbomolecular pumps (T2 and T3). The detection chamber housing a double-stack microchannel plate detector (13) is pumped using a $220 \mathrm{I} \mathrm{s}^{-1}$ turbomolecular pump (T4). An additional CMOS camera (C2) is located to observe fluorescence on a phosphor screen mounted at the back of the MCP plates. 
Before the liquid passes through the HPLC pump, it passes through a $10 \mu \mathrm{m}$ suction filter within the reservoir and after the HPLC pump it passes through a $2 \mu \mathrm{m}$ filter in the PEEK tubing. These filters help to prevent microparticles blocking the nozzle. The quartz nozzle is mounted on a motor-controlled $x y z$-positioning stage assembly for precise alignment of the liquid-microjet. For a $20 \mu \mathrm{m}$ diameter nozzle and aqueous solutions, typical flow rates and backing pressures are $0.6 \mathrm{ml} \mathrm{min}^{-1}$ and 70 bar. For water/methanol mixtures, we found lower flow rates were required to maintain a backing pressure within the upper limits of the HPLC pump, as expected because of the higher viscosity of methanol compared to water. ${ }^{37}$ The liquidmicrojet flows laminarly for around $2-4 \mathrm{~mm}$ before breaking up into a stream of droplets and the laser-liquid interaction occurs $\leq 1 \mathrm{~mm}$ below the nozzle in the laminar flow region. The liquid is caught into a recirculating system.

The recirculating system, based on one reported previously, ${ }^{38}$ is shown in detail in the expanded region of Fig. 1. It comprises a catcher assembly mounted on a motor-controlled $x y z$-positioning stage $3 \mathrm{~mm}$ below the laser-liquid interaction region. The liquid enters the catcher through a $500 \mu \mathrm{m}$ hole at the top of an electrically grounded beryllium copper cone tip $\left(\mathrm{CuBe}_{2}\right)$. Beryllium copper is a nonmagnetic alloy that is a good conductor of heat; this allows it to be used in the magnetic bottle spectrometer and to be maintained at a temperature of $40^{\circ} \mathrm{C}$ to ensure that the liquid does not freeze. Beryllium also forms a conducting oxide layer after contact with the liquid which ensures that the cone tip remains electrically grounded. The solution passes through antistatic explosion-proof laboratory tubing (BOLA), which is conductive and grounded to prevent electrostatic charging, and then squeezed through Tygon E-3603 soft tubing by a peristaltic recycling pump (Microliquids Recycling Pump Type 1) and returned to the reservoir connected to the liquid-microjet.

A liquid nitrogen cold-trap for liquid collection can be used instead of the recirculating system. The cold-trap is mounted on the same flange as the catcher assembly, directly below the liquidmicrojet. The trap is made of stainless steel with a copper inner and has a capacity of $0.5 \mathrm{l}$. The orifice is $4 \mathrm{~cm}$ in diameter, which results in an increase in pressure in the source chamber to $1-2 \times 10^{-4}$ mbar when the liquid-microjet is running, compared with $2 \times 10^{-5}$ mbar using the recirculating system. Cold traps with smaller orifices $(1.5 \mathrm{~cm})$ accompanied by additional diffusion pumping of the cold trap region have been shown to reduce the base pressure of the source chamber to between $10^{-4}$ and $10^{-5}$ mbar.

\section{B. Magnetic bottle photoelectron spectrometer}

A magnetic bottle time-of-flight spectrometer design was implemented because the magnetic fields increase the photoelectron detection efficiency dramatically, compared to a magnetic fieldfree design, but still allow the whole range of photoelectron kinetic energies to be collected in a single measurement. ${ }^{29,40-42}$ It is also relatively straightforward to achieve resolutions of $\Delta E / E \sim 1 \%$ in time-of-flight (TOF) photoelectron spectrometers. The magnetic bottle is formed by combining a strong permanent magnet generating a strong inhomogeneous magnetic field of around $1 \mathrm{~T}$ in the laser-liquid interaction region with a solenoid to generate a weak homogeneous magnetic field of $2 \mathrm{mT}$ along a drift tube. Photoelectrons emitted from the liquid after interaction with the laser are guided along the drift tube toward a microchannel plate (MCP) detector, where their arrival times are recorded relative to the trigger of the laser pulse. The design of our magnetic bottle photoelectron spectrometer is based on that of Neumark and coworkers. ${ }^{22}$ The strong permanent magnet is assembled from two magnetized cylinders of $\mathrm{Sm}_{2} \mathrm{Co}_{17}$, each with a diameter of $25 \mathrm{~mm}$ and length $15 \mathrm{~mm}$. A $15 \mathrm{~mm}$ long soft iron cone $\left(42^{\circ}\right.$ half angle) is placed in front of these to increase the field strength in the interaction region. The permanent magnet assembly is mounted on a vacuum compatible, motor-controlled, $x y z$-positioning stage (mechOnics MS30) for precise alignment of the magnetic bottle. The tip of the soft iron cone is located approximately $1 \mathrm{~mm}$ from the liquid-microjet and $2 \mathrm{~mm}$ from a $300 \mu \mathrm{m}$ gold-coated copper skimmer mounted on the front of a top-hat adapter. The skimmer acts as a differential pumping aperture between the interaction and time-of-flight chambers and is in a fixed position. The magnet tip is aligned with the center of the skimmer. The small orifice of the skimmer ensures that low pressures are maintained in the time-of-flight and detection chambers while the liquid-microjet is running $\left(5 \times 10^{-7}\right.$ and $6 \times 10^{-7}$ mbar, respectively), which is essential both for minimizing electron scattering and for the high-voltage operation of the MCP detector. A CMOS (complementary metaloxide semiconductor) camera (C1, Fig. 1) with a resolution of 1.92 MPix (iDS, UI-3250LE) is mounted outside the interaction chamber and is positioned to visualize the liquid-microjet during operation (Sec. III B).

The solenoid of the magnetic bottle is formed by wrapping $2 \mathrm{~mm}$ diameter Kapton-insulated copper wire with approximately 394 turns per meter around a $666 \mathrm{~mm}$ long polytetrafluoroethylene (PTFE) sleeve outside the time-of-flight tube. The solenoid begins $41 \mathrm{~mm}$ from the interaction region and ends $14 \mathrm{~mm}$ beyond the MCP detector. The solenoid current is kept at $4 \mathrm{~A}$. An outer PTFE tube isolates the solenoid and is surrounded by two $0.5 \mathrm{~mm}$ thick $\mu$-metal tubes separated by $5.85 \mathrm{~mm}$ to shield the time-of-flight tube from external magnetic fields. A drift tube of length $630 \mathrm{~mm}$ is installed inside the time-of-flight tube and is perforated with $3 \mathrm{~mm}$ diameter holes to allow it to be pumped efficiently.

Electrons that reach the end of the flight tube are detected by a double stack of MCPs with a phosphor screen mounted behind them (Beam Imaging Solutions, BOS-25). A copper mesh with $90 \%$ transmission, grounded to the drift tube, is placed in front of the MCPs to accelerate the electrons before they hit the detector. Currently, the front surface of the MCPs is grounded, but a voltage can be applied to further accelerate the electrons if needed. The electron signal is amplified by applying a voltage of $+1800 \mathrm{~V}$ to the back MCP. These amplified electrons are projected onto the phosphor screen by applying a potential of 2000-3400 V between the back of the MCPs and the conductive layer of the phosphor screen. The image of the photoelectrons hitting the MCP on the phosphor screen is visualized using a CMOS camera (C2, Fig. 1; iDS, UI-1220LE) and assists spatial alignment in the interaction chamber (Sec. III B). The photoelectron current is capacitively decoupled from the phosphor screen, amplified (20 V/V; Ortec 9326) and recorded, together with arrival time relative to the trigger of the laser pulse, by a high-speed digitizer (Keysight U5309A) using a typical bin width of 500 ps. The digitized electron signal associated with each laser pulse is then discriminated and the electron time-of-flight distribution determined using LabVIEW based software. 


\section{INSTRUMENT OPERATION}

To determine the relationship between the photoelectron timeof-flight (TOF) and photoelectron kinetic energy (eKE), multiphoton ionization (MPI) spectra of gaseous NO are recorded. Resonance-enhanced MPI (REMPI) spectra of gaseous Xe are recorded to determine the energy resolution, to optimize the alignment of the components in the interaction region and to determine the streaming potential of the liquid-microjet. Gas samples are admitted through a $100 \mu \mathrm{m}$ nozzle, held approximately $4 \mathrm{~mm}$ behind the laser-liquid interaction region and angled so that the nozzle is clear of the path of the laser light and CMOS camera (C1) monitoring the interaction region.

Femtosecond laser pulses are generated by frequency upconverting the output of an optical parametric amplifier (TOPAS) pumped by an amplified Ti:Sapphire femtosecond laser system (Coherent Micra and Legend) operating at $1 \mathrm{kHz}$. The photon flux is attenuated so that the electron count-rates are typically $500 \mathrm{~Hz}$ (below 1 photoelectron per pulse) to avoid space-charge effects and saturation of the detector.

The procedure for operation is as follows. (1) Two TOF calibrations using NO gas are performed in the absence of the liquidmicrojet nozzle (Sec. III A); one of these is with the magnet in the optimum magnetic bottle geometry, for sample measurements, and the other is with the magnet translated away from the interaction region, for streaming potential measurements. (2) With each NO calibration, a photoelectron spectrum of Xe is also recorded to check the accuracy of the TOF calibration and to measure the energy offset that accounts for stray electric fields arising from the liquid-microjet nozzle (Sec. III D). (3) The sample solution is run continuously through the liquid-microjet at ambient pressure for around $24 \mathrm{~h}$ to passivate the system (Sec. III C). (4) The liquid-microjet is placed in the spectrometer and the pressures of the chambers are gradually lowered (Sec. III B). (5) The liquidmicrojet and recirculator are aligned to the laser focus and the skimmer orifice (Sec. III B). (6) A streaming potential measurement is made with the magnet translated away from the interaction region (Sec. III D). (7) Photoelectron spectra of the sample are recorded with the magnet in the optimum magnetic bottle geometry. (8) If time permits, a second streaming potential measurement is performed.

\section{A. Time-of-flight calibration}

Photoionization with total photon energy $h v>9.24 \mathrm{eV}$ gives rise to transitions to vibronic states of $\mathrm{NO}^{+}: \mathrm{NO}\left(X^{2} \Pi_{1 / 2}, v^{\prime \prime}=0\right)$ $\rightarrow \mathrm{NO}^{+}\left(X^{1} \Sigma^{+}, v^{+}\right)$. Photoelectron spectra of NO following MPI at $268.5 \mathrm{~nm}(4.62 \mathrm{eV}), 244.3 \mathrm{~nm}(5.08 \mathrm{eV}), 240.0 \mathrm{~nm}(5.09 \mathrm{eV})$, $238.2 \mathrm{~nm}(5.17 \mathrm{eV})$, and $238.0 \mathrm{~nm}(5.21 \mathrm{eV})$, recorded in the absence of the liquid-microjet nozzle, are presented in Fig. 2. Six vibronic bands $\left(v^{+}=0-5\right)$ are observed following three-photon ionization at $268.5 \mathrm{~nm}$, with TOFs in the range 540-650 ns. Nine vibronic bands $\left(v^{+}=0-2\right)$ are observed following two-photon ionization at $244.3 \mathrm{~nm}, 240.0 \mathrm{~nm}$, and $238.0 \mathrm{~nm}$, with TOFs in the range 850-1200 ns. The electron kinetic energies corresponding to the vibronic transitions are determined using $\mathrm{eKE}=n h v-\operatorname{IE}\left(v^{+}\right)$, where $n$ is the number of photons in the photoionization process and $\operatorname{IE}\left(v^{+}\right)$is the ionization energy for $\mathrm{NO}\left(X^{2} \Pi_{1 / 2}, v^{\prime \prime}=0\right) \rightarrow$ $\mathrm{NO}^{+}\left(X^{1} \Sigma^{+}, v^{+}, N^{+}=0\right){ }^{43}$ The maxima of the peaks in the TOF

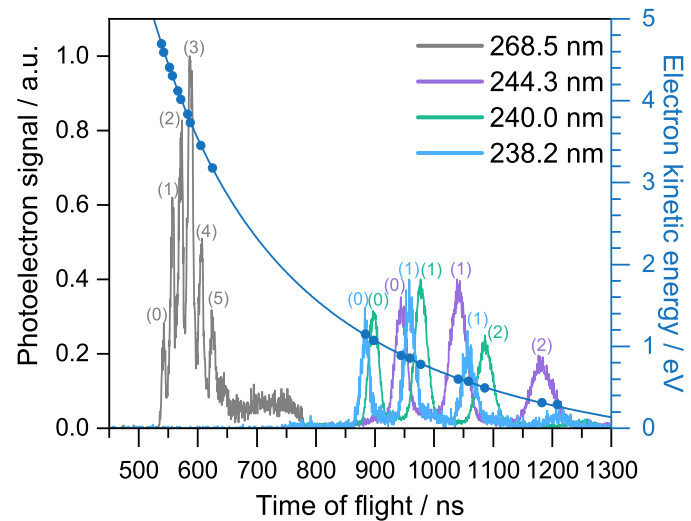

FIG. 2. Time-of-flight photoelectron spectra of NO recorded following MPI with $268.5 \mathrm{~nm}$ (4.62 eV, gray), $244.3 \mathrm{~nm}$ (5.08 eV, purple), $240.0 \mathrm{~nm}$ (5.17 eV, green), and $238.2 \mathrm{~nm}(5.21 \mathrm{eV}$, blue) femtosecond laser pulses. The electron kinetic energies for $\mathrm{NO}\left(X^{2} \Pi_{1 / 2}, v^{\prime \prime}=0\right) \rightarrow \mathrm{NO}^{+}\left(X^{1} \Sigma^{+}, v^{+}=0-5\right)$ vibronic transitions were determined using known ionization potentials ${ }^{43}$ and assigned to the peaks in the time-of-flight spectra (circles) and fitted to Eq. (1) (dark blue curve). Numbers in brackets represent values of $v^{+}$

spectra are fitted to

$$
\mathrm{eKE}=\frac{m_{e}}{2}\left(\frac{s}{t-t_{0}}\right)^{2}-E_{0},
$$

where $\mathrm{eKE}$ is the electron kinetic energy associated with each observed vibronic transition, $t$ is the TOF, $m_{e}$ is the electron mass, $s$ is the distance from the laser-liquid interaction point to the detector, $t_{0}$ is a temporal offset that accounts for the time delay between the trigger to the digitizer, and the measured electron arrival time and $E_{0}$ is an energy offset that accounts for any stray electric fields that affect the velocity of the photoelectrons. The calibration constants $s, t_{0}$, and $E_{0}$ are obtained from the fit and for the calibration in Fig. 2 are $s=0.65 \pm 0.01 \mathrm{~m}, t_{0}=64.88 \pm 5.48 \mathrm{~ns}$, and $E_{0}=0.65 \pm 0.02 \mathrm{eV}$. The error in $t_{0}$ corresponds to an error of $\pm 0.07 \mathrm{eV}$. The calibration constants are dependent on the relative alignment of the liquid-microjet, laser beam, and magnetic bottle, and so they are determined daily.

The calibration fitting parameters allow electron TOF to be converted to electron kinetic energy (eKE). Photoelectron spectra are then extracted from the TOF spectra by multiplying the photoelectron counts by the Jacobian $m_{e} s^{2} /\left(t-t_{0}\right)^{3}$. It is worth noting that a value of $\left|E_{0}\right|>0$ requires that the Jacobian transformation is performed before accounting for $E_{0}$.

A similar procedure is used to calibrate the spectrometer with the magnet translated away from the interaction region, with the geometry used for streaming potential measurements.

The energy resolution is determined from photoelectron spectra of Xe recorded following $2+1$ REMPI via the $5 \mathrm{p}^{5}\left({ }^{2} \mathrm{P}_{3 / 2}\right) 6 \mathrm{p}[1 / 2]_{0}$ state to $\mathrm{Xe}^{+}\left({ }^{2} \mathrm{P}_{3 / 2}\right)$ and $\mathrm{Xe}^{+}\left({ }^{2} \mathrm{P}_{1 / 2}\right)$ (Fig. 3). $\Delta E / E$ is $2 \%-3 \%$ when the magnetic is in the optimum magnetic bottle geometry for sample measurements and is limited by the bandwidth of the femtosecond lasers.

Photoelectron spectra of Xe recorded for the two magnetic bottle geometries are compared in Figs. 3(b) and 3(c). When the magnet is very close to the interaction region, all photoelectrons emitted in the $2 \pi$ solid angle facing the detector are collected. ${ }^{40}$ When the magnet is translated a fraction of a millimeter away from the interaction 


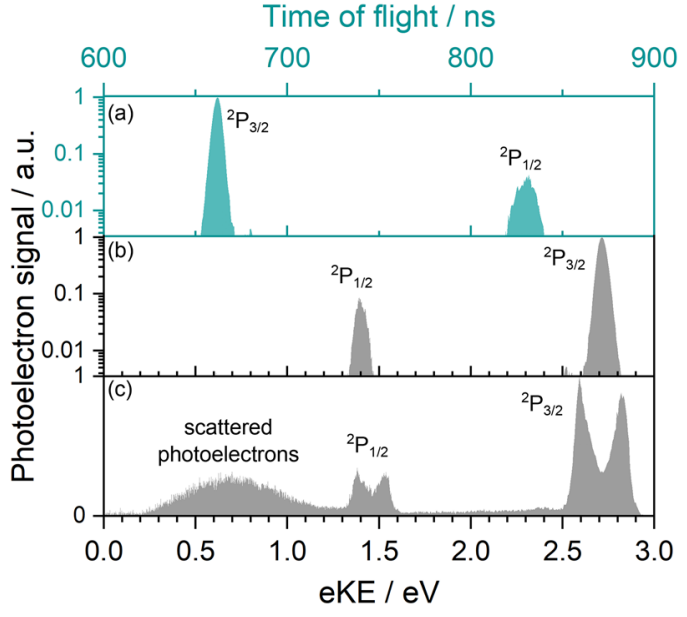

FIG. 3. $2+1$ REMPI photoelectron spectra of gaseous $X e$ recorded at $249.7 \mathrm{~nm}$ : $\mathrm{Xe}\left({ }^{1} \mathrm{~S}_{0}\right) \rightarrow \mathrm{Xe}{ }^{+}\left({ }^{2} \mathrm{P}_{3 / 2}\right) / \mathrm{Xe}^{+}\left({ }^{2} \mathrm{P}_{1 / 2}\right)$ via the $5 \mathrm{p}^{5}\left({ }^{2} \mathrm{P}_{3 / 2}\right) 6 \mathrm{p}[1 / 2]_{0}$ intermediate. Photoelectron spectra are plotted as a function of TOF (a) and eKE (b), on logarithmic scales to emphasize the lower intensity feature attributed to $\mathrm{Xe}\left({ }^{1} \mathrm{~S}_{0}\right) \rightarrow \mathrm{Xe}^{+}\left({ }^{2} \mathrm{P}_{1 / 2}\right)$. (c) Photoelectron spectrum plotted as a function of eKE following translation of the magnet away from the laser-microjet interaction region for streaming potential measurements (Sec. III D), illustrating the split peaks discussed in the main text.

region, photoelectrons that are emitted in the $2 \pi$ solid angle facing away from the detector are turned around when they try to enter the region of higher magnetic field. This manifests itself as an increase in detection efficiency and a slight increase in the spread of arrival times. For the photoelectron spectra presented in Fig. 3(b), the magnet position was set for such maximum photoelectron signal. As the magnet is moved further away from the interaction region, the spread of arrival times continues to increase and a dip appears in the center of the photoelectron signal [Fig. 3(c)]. This dip is attributed to the forward and backward photoelectron signals separating and a drop in the efficiency of collection of photoelectrons emitted with a significant component of their velocity perpendicular to the TOF axis. For our calibration of the spectrometer with the magnet translated away from the interaction region, we measure the positions of the centers of the split peaks. We attribute the broad feature in Fig. 3(c) extending from 0.2 to $1.3 \mathrm{eV}$ to background photoelectrons from scattered light and suspect that it is only observed when the magnet is translated away from the interaction region because of the associated increase in collection volume.

\section{B. Liquid-microjet alignment}

The liquid-microjet is aligned to be directly between the skimmer orifice and the tip of the soft iron cone on front of the magnet (magnet tip). The laser beam intersects the liquid-microjet orthogonal to the axes of the liquid-microjet and the skimmer-magnet tip. The catcher assembly is mounted directly below the liquid-microjet. Operation begins with aligning the running liquid-microjet with the catcher at ambient pressure in the interaction chamber, while the time-of-flight and detection chambers are kept at lower pressures by continuous pumping with the backing pump for T4 [Fig. 1]. During this initial alignment, the liquid-microjet is kept far enough away from the skimmer to avoid splashes of liquid entering the time-of-flight chamber.

The interaction chamber pressure is then lowered to around $10^{-3}$ mbar using rotary and cryogenic pumping before turning on the turbomolecular pump to reduce the pressure further. At this point, the pressure reading provides another means of monitoring the alignment; if the liquid comes into contact with the tip of the catcher, splashes cause the pressure reading in the interaction chamber to increase.

Once the liquid-microjet is aligned with the catcher tip and the pressure in the interaction chamber is around $2 \times 10^{-5} \mathrm{mbar}$, the liquid-microjet and catcher assembly are translated together until they are approximately $1 \mathrm{~mm}$ from the skimmer. Crude alignment is achieved by monitoring the pressure in the time-of-flight chamber; when the jet is directly in front of the skimmer, the pressure passes through a maximum. The alignment is refined by monitoring the spatial distribution of photoelectrons on the phosphor screen behind the MCPs using CMOS camera C2. ${ }^{29}$ First, gaseous Xe is ionized in a $2+1$ REMPI process at $249.7 \mathrm{~nm}$ without the liquidmicrojet running. This gives rise to an image of a line of photoelectrons corresponding to electrons emitted along the path of the laser beam; the maximum length of the line corresponds to the diameter of the skimmer. This allows us to draw an image of the circumference of the skimmer orifice. Next, photoelectrons are generated from the liquid-microjet which gives rise to an image of a point source of electrons corresponding to the laser-liquid-microjet interaction. When the laser-liquid-microjet interaction region is correctly aligned with the center of the skimmer, the image of the point source of electrons sits in the center of the image of the skimmer orifice.

\section{Solution preparation}

To ensure solutions of interest are free from microparticles, all glassware is cleaned using aqua regia. The solutions are degassed using an ultrasonic cleaner and filtered through a $0.45 \mu \mathrm{m}$ filter (Whatman Spartan 30).

\section{Streaming potential}

The liquid-microjet is inherently electrically charged due to dynamic separation of the electrical double layers formed around the inner walls of the quartz nozzle. The resulting streaming current generates a streaming potential that can accelerate or decelerate photoelectrons emitted from the liquid-microjet. ${ }^{45}$ The sign and amplitude of the streaming potential depend on the solution and its concentration, the nozzle diameter, and the flow-rate. ${ }^{46}$ To minimize the streaming potential, electrolytes are added to the solution. ${ }^{9,23,46-52}$ We have also found that the addition of electrolytes is useful for grounding the liquid-microjet when using our recirculating system (see below).

The streaming potential can vary significantly by a few tenths of an electron volt as a result of the time it takes for the ionexchange processes that are responsible for the streaming potential to reach equilibrium. ${ }^{15,16,44}$ Using a method similar to that employed by Kurahashi et al., ${ }^{15}$ we found that the current becomes stable after around $24 \mathrm{~h}$. Thus, before recording liquid-microjet photoelectron spectra, we run the solution through the nozzle for at least $24 \mathrm{~h}$ to 
passivate it. Following this procedure, we found that the streaming potential varied by less than $0.02 \mathrm{eV}$ before and after liquid-microjet photoelectron spectroscopy measurements.

To quantify the streaming potential, we measure it using a method similar to the one reported by Tang et al. ${ }^{12}$ The magnet is translated away from the skimmer and $2+1$ REMPI photoelectron spectra of gaseous Xe at $249.7 \mathrm{~nm}$ are recorded with the liquid-microjet running at various distances between the ionization point and the magnet. Photoelectron eKE is plotted as a function of distance $x$ (Fig. 4). eKE $(x)$ can be expressed as ${ }^{12}$

$$
\mathrm{eKE}(x)=\mathrm{eKE}_{\text {true }}-\frac{L \phi_{\text {str }}}{L+x}+V
$$

where $\operatorname{eKE}(x)$ is determined using a TOF to eKE calibration (Sec. III A) carried out with the magnetic bottle geometry set for streaming potential measurements, $\mathrm{eKE}_{\text {true }}=3 h v-\mathrm{IE}$ where IE is the known ionization energy of $\mathrm{Xe}, L$ is the distance between the ionization point and the skimmer, $x$ is the distance between the ionization point and the liquid-microjet, $\phi_{\text {str }}$ is the streaming potential, and $V$ accounts for additional fields in the magnetic bottle photoelectron spectrometer with the liquid-microjet nozzle in place. ${ }^{17} \mathrm{~L}$ is determined using CMOS camera $\mathrm{C} 1$ that monitors the interaction region. The pixels of the camera are scaled by translating the liquid-microjet a complete turn on the micrometer, which is equivalent to $1 \mathrm{~mm}$. This distance in pixels sets a unit measurement for millimeters, which allows $L$ to be determined from the number of pixels between the camera images of the skimmer orifice and the liquid-microjet. To measure a change in magnitude of the streaming potential as the liquid-microjet is moved away from the ionization point, we find that a total translation distance $x \sim 5 \mathrm{~mm}$ is required. To allow for this, the magnet is translated to a distance of around $8 \mathrm{~mm}$ from the ionization point.

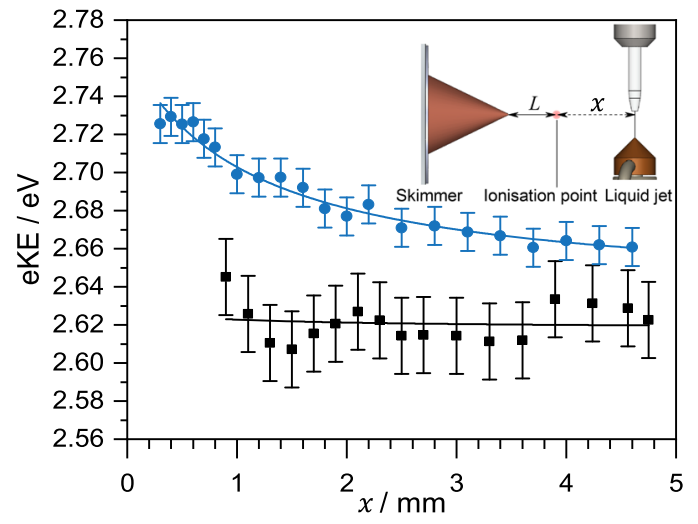

FIG. 4. Photoelectron eKE measured following $2+1$ REMPI of Xe at $249.7 \mathrm{~nm}$ in the presence of a liquid-microjet of $100 \mathrm{mM}$ phenol and $30 \mathrm{mM} \mathrm{NaF}$ and plotted as a function of distance $x$ between the ionization point and the liquid-microjet. Measured eKEs correspond to the $\mathrm{Xe}\left({ }^{1} \mathrm{~S}_{0}\right) \rightarrow \mathrm{Xe}^{+}\left({ }^{2} \mathrm{P}_{3 / 2}\right)$ ionization process. Blue circles represent measurements made using a liquid nitrogen cold-trap ( $L$ $=1.10 \mathrm{~mm}, \phi_{\text {str }}=-0.13 \pm 0.01 \mathrm{eV}$ ). Black squares represent measurements made using the recirculating system $\left(L=1.05 \mathrm{~mm}, \phi_{\text {str }} \sim 0\right.$; see the text). Error bars represent the mean maximum deviations in $\operatorname{eKE}(x)$ from the fitted lines from two separate data sets. Inset: experimental geometry used for streaming potential measurements.
Equation (2) implies that both $\phi_{\text {str }}$ and $V$ can be determined from the measurement; however, we find that only $\phi_{\text {str }}$ can be determined accurately because of the peak splitting discussed in Sec. III A. To determine $\phi_{\text {str }}$ accurately requires $\operatorname{eKE}(x)$ to be determined accurately, which is challenging as it varies by less than $0.1 \mathrm{eV}$ (Fig. 4). Figure 5 shows Xe spectra measured at four distances of the liquid-microjet from the ionization point. It is clear that an accurate measurement of the center of the splitting is more difficult when the liquid-microjet is closer to the ionization point. The peak at lower eKE $(\sim 2.44 \mathrm{eV})$ is significantly broader in Fig. 5(a) compared to Fig. 5(d), whereas the peak at higher eKE has at a fairly constant width. Thus, we choose to fit a Gaussian to the higher eKE feature to determine the streaming potential, which depends only on the curvature of $\operatorname{eKE}(x)$. Consequently, $V$ cannot be extracted from these measurements. Instead, we determine $V$ in a separate measurement which is described later in this section.

Streaming potential measurements for $100 \mathrm{mM}$ aqueous phenol and $30 \mathrm{mM} \mathrm{NaF}$ (as the electrolyte) using our recirculating system and a cold trap are compared in Fig. 4. The cold-trap data shows that the eKE increases when the liquid-microjet is closer to the ionization point, implying that the liquid-microjet is negatively charged. Fitting the data presented in Fig. 4 to Eq. (2) gives $\phi_{\text {str }}=-0.13 \pm 0.01 \mathrm{eV}$ (blue curve). The magnitude of $\phi_{\text {str }}$ is similar to measurements reported using other liquid-microjet photoelectron spectrometers with cold traps; $\left|\phi_{\text {str }}\right|=0.1-0.3 \mathrm{eV}$ for aqueous and alcohol solutions containing electrolytes. ${ }^{14-17,23}$

As $x \rightarrow 0, \operatorname{eKE}(x) \rightarrow \mathrm{eKE}_{\text {true }}-\phi_{\text {str }}+V$, and as $x$ becomes very large, $\mathrm{eKE}(x) \rightarrow \mathrm{eKE}_{\text {true }}+V$. The true eKEs of cold-trap data measurements are therefore obtained from the measured eKEs, after $V$ has been determined, using

$$
\mathrm{eKE}_{\text {true }}=\mathrm{eKE}_{\text {meas }}+\phi_{\text {str }}-V
$$
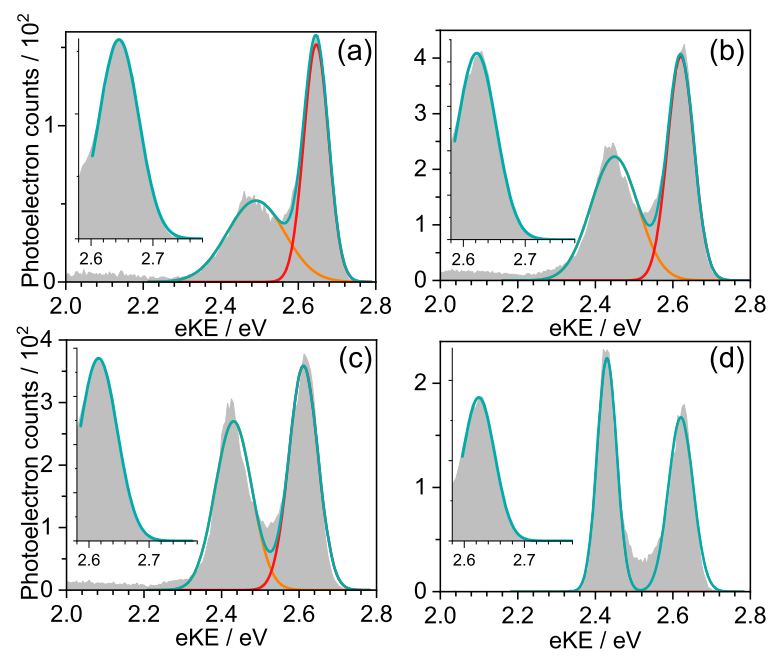

FIG. 5. Photoelectron spectra following $2+1$ REMPI of $X e$ at $249.7 \mathrm{~nm}$ recorded during the measurement of the streaming potential at various distances of the liquid-microjet from the ionization point, $x$. Fits with two and one (inset) Gaussians are shown. $x=$ (a) $0.9 \mathrm{~mm}$, (b) $1.9 \mathrm{~mm}$, (c) $3.0 \mathrm{~mm}$, and (d) $4.75 \mathrm{~mm}$. 
For the recirculating system, $\operatorname{eKE}(x)$ is a straight line (Fig. 4), indicating that the streaming potential is effectively zero. We believe this can be attributed to the electrically conducting catcher tip grounding the liquid-microjet and suspect that the fluctuations in the data in Fig. 4 may arise from the solution not being in constant contact with the grounded catcher tip. Setting $\phi_{\text {str }}=0$ in Eq. (2), gives the true eKEs of recirculating data measurements obtained from the measured eKEs by

$$
\mathrm{eKE}_{\text {true }}=\mathrm{eKE}_{\text {meas }}-V .
$$

Both Eqs. (1) and (2) have constant terms accounting for additional fields, $E_{0}$ and $V$, respectively. $V$ is an additional energy offset introduced by having the liquid-microjet nozzle in the spectrometer. To determine $V$, we recorded photoelectron TOF spectra following $2+1$ REMPI of Xe at $249.7 \mathrm{~nm}$ with the magnetic bottle geometry set for streaming potential measurements, first without the liquid-microjet nozzle in place and then with the liquid-microjet nozzle in place and the liquid-microjet running. We then used the energy difference between $\mathrm{Xe}^{+}\left({ }^{2} \mathrm{P}_{3 / 2}\right)$ and $\mathrm{Xe}^{+}\left({ }^{2} \mathrm{P}_{1 / 2}\right)(1.31 \mathrm{eV})$ to calibrate the TOF to eKE conversion. Figure 6 shows the fits of the two TOF spectra using Eq. (1) for both the recirculator [Fig. 6(a), without liquid-microjet nozzle; Fig. 6(b), with liquid-microjet running] and cold-trap measurements [Fig. 6(c), without liquid-microjet nozzle; Fig. 6(d), with liquid-microjet running]. The data points are at the approximate centers of the peak splittings and the error bars reflect the uncertainty in these measurements. The distance parameter $s$ is fixed at the value determined accurately from our usual calibration procedure (Sec. III A), and the energy offset parameter $E_{0}$ is varied to optimize the fit through the two data points (green lines).

For the cold-trap, $E_{0}=0.57 \pm 0.01 \mathrm{eV}$ and $0.48 \pm 0.03 \mathrm{eV}$ for measurements without the liquid-microjet nozzle and with the liquid-microjet running, respectively. The difference between these measurements is $-0.09 \pm 0.03 \mathrm{eV}$ and corresponds to $V$. For the recirculator, $E_{0}=0.58 \pm 0.01 \mathrm{eV}$ and $0.52 \pm 0.03 \mathrm{eV}$ for measurements without the liquid-microjet nozzle and with the liquidmicrojet running, respectively. The difference between these measurements, $V=-0.06 \pm 0.03 \mathrm{eV}$. These values of $V$ each lie within the error bars of the other, as expected since $V$ is the energy offset introduced by the liquid-microjet nozzle.

\section{INSTRUMENT PERFORMANCE}

To demonstrate the performance of our recirculating liquidmicrojet photoelectron spectrometer, we present a comparison between $275 \mathrm{~nm}$ REMPI photoelectron spectrum of $100 \mathrm{mM}$ phenol in aqueous solution (with $30 \mathrm{mM} \mathrm{NaF}$ as an electrolyte) recorded using the recirculating system and the liquid nitrogen cold trap (Fig. 7).

Phenol absorbs radiation around $270 \mathrm{~nm}(4.6 \mathrm{eV})$ and $205 \mathrm{~nm}$ $(6.0 \mathrm{eV})$ in aqueous solutions; these bands arise from transitions from the electronic ground state, $S_{0}$, to $1^{1} \pi \pi^{*}$ and $2^{1} \pi \pi^{*}$ states. Between these two ${ }^{1} \pi \pi^{*}$ states, around $235 \mathrm{~nm}(5.28 \mathrm{eV})$, is a dissociative $1^{1} \pi \sigma^{*}$ state. In a recent $1+1$ MPI photoelectron spectroscopy study of the electronic structure of aqueous phenol using our liquidmicrojet photoelectron spectrometer with the liquid nitrogen coldtrap, we found that for wavelengths in the range $275-250 \mathrm{~nm}$, the $1+1$ photoelectron spectra were best fit with single Gaussians
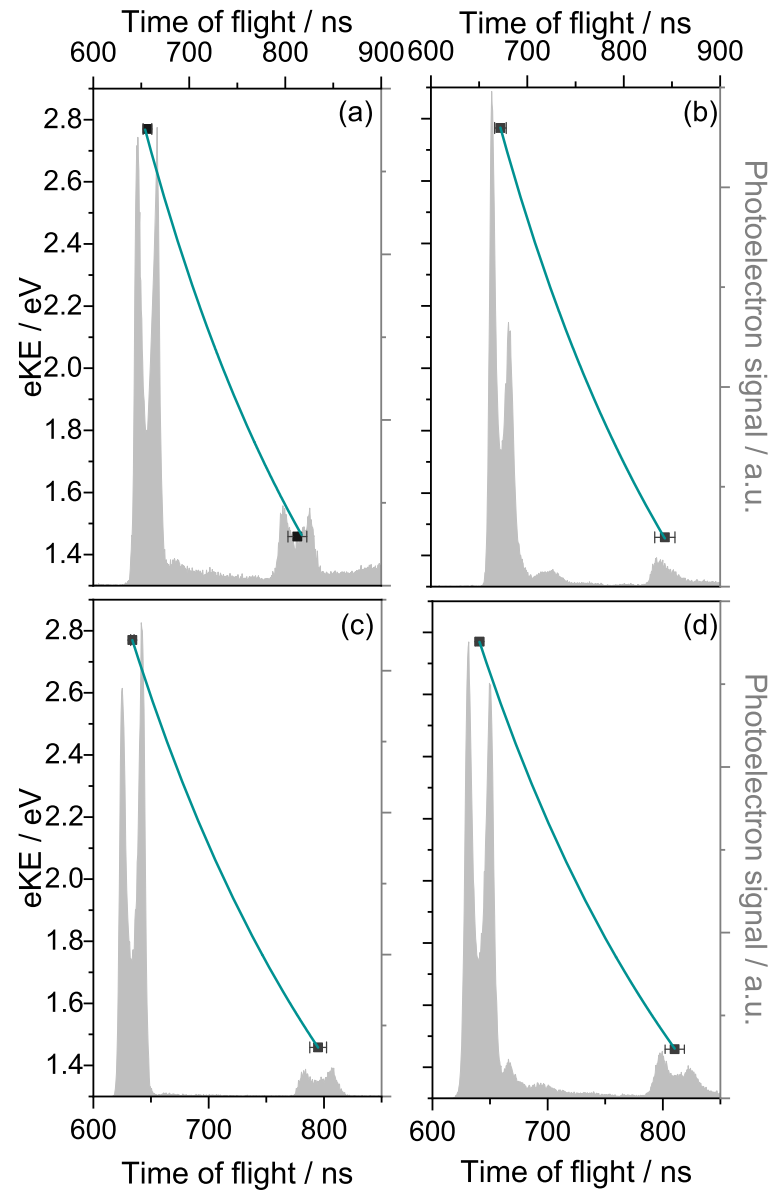

FIG. 6. Photoelectron TOF spectra following $2+1$ REMPI of Xe at $249.7 \mathrm{~nm}$ with the magnet translated away from the interaction region, for streaming potential measurements. [(a) and (b)]: recirculator data (a) without the liquid-microjet nozzle and (b) with the liquid-microjet nozzle $4.75 \mathrm{~mm}$ from the ionization point with the jet running. [(c) and (d)]: cold-trap data (c) without the liquid-microjet nozzle and (d) with the liquid-microjet nozzle $4.75 \mathrm{~mm}$ from the ionization point with the jet running. Fitting the approximate centers of the peak splittings to Eq. (1) (green lines) gives $E_{0}=0.58 \pm 0.01 \mathrm{eV}(\mathrm{a}), 0.52 \pm 0.03 \mathrm{eV}(\mathrm{b}), 0.57 \pm 0.03 \mathrm{eV}$ (c), and $0.48 \pm 0.03 \mathrm{eV}(\mathrm{d})$. The difference between measurements of $E_{0}$ from (a) and (b) corresponds to $V=-0.06 \pm 0.03 \mathrm{eV}$ for the recirculator measurements and the difference between measurements of $E_{0}$ from (c) and (d) corresponds to $V=-0.09 \pm 0.03 \mathrm{eV}$ for the cold-trap measurements

with maxima around $3.5 \pm 0.1 \mathrm{eV}$ eBE, corresponding to the $1^{1} \pi \pi^{*}$ $-\mathrm{D}_{0}$ vertical ionizations energy (VIE). ${ }^{35}$ At $235.5 \mathrm{~nm}$, where the UV absorption cross section is a minimum, we found that direct ionization from $\mathrm{S}_{0}$ gave rise to two Gaussians centered around $2.9 \pm 0.1 \mathrm{eV}$ and $2.0 \pm 0.1 \mathrm{eV}$ eKE, corresponding to $7.6 \pm 0.1 \mathrm{eV}$ and $8.5 \pm 0.1 \mathrm{eV}$ $\mathrm{S}_{0}-\mathrm{D}_{0}$ and $\mathrm{S}_{0}-\mathrm{D}_{1}$ VIEs, respectively. These VIEs are in agreement with those measured using XUV radiation at BESSY II (7.8 $\pm 0.1 \mathrm{eV}$ and $8.6 \pm 0.1 \mathrm{eV}){ }^{50}$ Recently, Roy et al. reported a $267 \mathrm{~nm}$ $1+1$ REMPI photoelectron spectroscopy study of aqueous phenol using a liquid-microjet photoelectron spectrometer with a cold trap. Their spectrum, plotted as a function of binding energy, was fit with two Gaussians from which they determined the VIEs from $S_{0}$ to be 


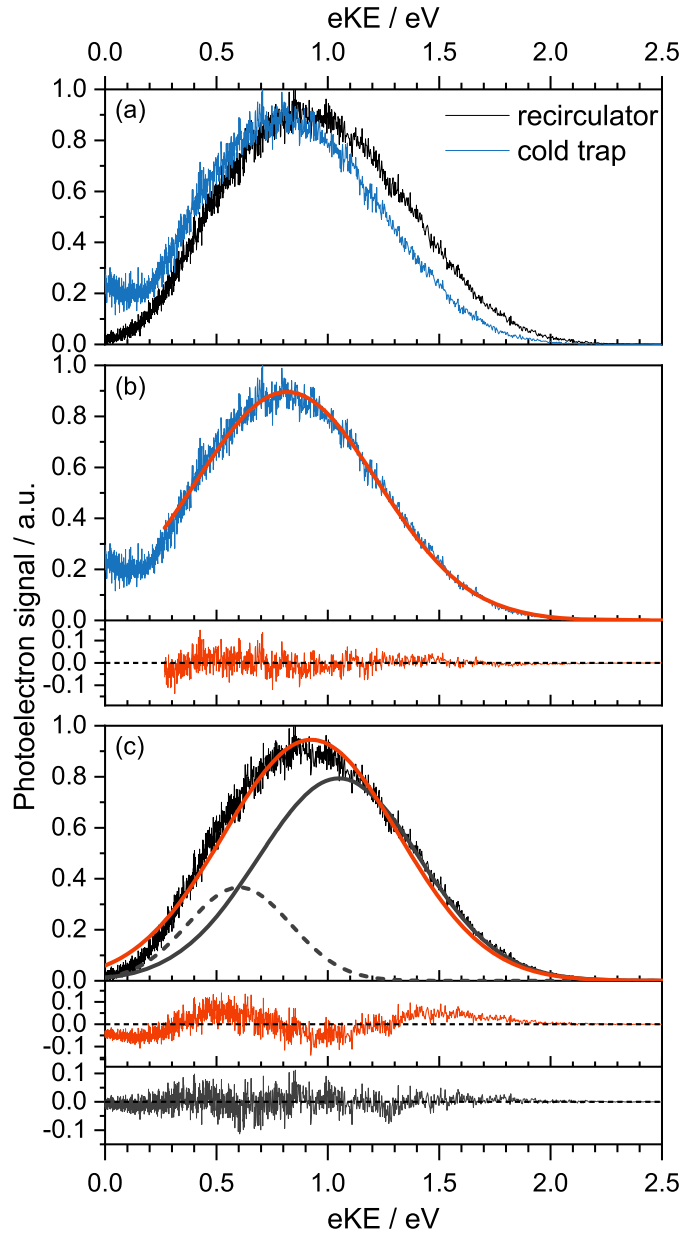

FIG. 7. Photoelectron eKE recorded following $1+1$ REMPI of $100 \mathrm{mM}$ aqueous phenol with $30 \mathrm{mM} \mathrm{NaF}$ at $275 \mathrm{~nm}$. (a) Comparison between photoelectron spectra recorded using the recirculator system (black) and the liquid nitrogen coldtrap (blue). (b) Photoelectron spectrum recorded using the liquid nitrogen cold-trap with a constrained single Gaussian fit (red) together with the residual (below the main panel); the fit is for eKE $>0.2 \mathrm{eV}$ to avoid the low eKE data affected by the increased vapor pressure in the interaction chamber when using the cold-trap. (c) Photoelectron spectrum recorded using the recirculator with a relaxed single Gaussian fit (red) and a two Gaussian fit (solid and dashed black lines); corresponding residuals are shown below the main panel for the single Gaussian fit (red) and the two-Gaussian fit (black). The solid black Gaussian is centered at $1.1 \pm 0.1 \mathrm{eV}$ and can be attributed to $1^{1} \pi \pi^{*}-D_{0}$ ionization and the dashed black Gaussian is centered at $0.6 \pm 0.1 \mathrm{eV}$ and can be attributed to $1^{1} \pi \pi^{*}-D_{1}$ ionization.

$8.0 \pm 0.1 \mathrm{eV}$ and $8.5 \pm 0.1 \mathrm{eV}$, which also agree well with the XUV measurements. ${ }^{28}$ Their analysis was based on two assumptions: (1) that the Franck-Condon overlap between the first ${ }^{1} \pi \pi^{*}$ state and $D_{0}$ is the same as that between $S_{0}$ and $D_{0}$ and (2) that inelastic scattering shifts the spectrum in a similar way to photoelectron spectra of solvated electrons in aqueous solution.

In Fig. 7(a), 275 nm $1+1$ REMPI photoelectron spectra of phenol recorded using our liquid-microjet photoelectron spectrometer with the liquid nitrogen cold-trap and the recirculator are compared. The cold-trap data has a noticeable background count at low eKE compared to the recirculator data, that we attribute to increased scattering of low eKE electrons from water vapor in the interaction chamber when operating with the cold-trap. We wish to point out that the cold-trap data presented here also has a higher background count than the data presented in our earlier work. ${ }^{35}$ This is due to an error in the way the Jacobian was implemented in the data analysis; fortuitously, it does not change the interpretation of the data within experimental error.

Like with our earlier work, the cold-trap data can be fit with a single Gaussian, centered at $0.8 \pm 0.1 \mathrm{eV}$ eKE [Fig. 7(b)]. This corresponds to $3.7 \pm 0.1 \mathrm{eV} 1^{1} \pi \pi^{*}-\mathrm{D}_{0}$ VIE, which is within the error bars of our earlier measurement. The recirculator data, however, is best fit with two Gaussians centered at $1.1 \pm 0.1 \mathrm{eV}$ (solid black line) and $0.6 \pm 0.1 \mathrm{eV}$ (dashed black line) eKE [Fig. 7(c)]. The feature at higher eKE $\left(1^{1} \pi \pi^{*}-D_{0}\right)$ corresponds to a VIE of $3.4 \pm 0.1 \mathrm{eV}$. The feature at lower eKE $\left(1^{1} \pi \pi^{*}-D_{1}\right)$ corresponds to a VIE of $3.9 \pm 0.1 \mathrm{eV}$. The lower eKE feature can only be extracted, with confidence, from our recirculator data because the reduced vapor pressure around the jet allows us to fit to very low eKEs. It is worth noting that the residual of the fit at low eKE is only $8 \%$ at $0.1 \mathrm{eV}$, highlighting the good transmission efficiency of our spectrometer at low eKE. It is also worth noting that the area of the peak corresponding to $1^{1} \pi \pi^{*}-$ $\mathrm{D}_{0}$ ionization is around three times larger than that of the area of the peak corresponding to $1^{1} \pi \pi^{*}-D_{1}$ ionization, in good agreement with our earlier calculations of photoionization cross sections from the $1^{1} \pi \pi^{*}$ state. ${ }^{35}$ This contrasts with the $267 \mathrm{~nm}$ MPI photoelectron spectrum reported by Roy et al. in which the area of the peak corresponding to $1^{1} \pi \pi^{*}-\mathrm{D}_{0}$ ionization was less than the area of the peak corresponding to $1^{1} \pi \pi^{*}-D_{1}$ ionization. ${ }^{28}$ If we were to assume that the VIEs from $S_{0}$ could be determined by adding the $\mathrm{S}_{0}-1^{1} \pi \pi^{*}$ adiabatic excitation energy, estimated as $4.46 \mathrm{eV}$ from the uv-vis absorption spectrum, ${ }^{35}$ to the VIEs from the $1^{1} \pi \pi^{*}$ state and that inelastic scattering shifts the spectrum in a similar way to photoelectron spectra of solvated electrons in aqueous solution, ${ }^{53}$ we would obtain $\mathrm{S}_{0}-\mathrm{D}_{0}$ VIEs of $7.6 \pm 0.1 \mathrm{eV}$ and $8.5 \pm 0.1 \mathrm{eV}$. These values agree with the BESSY II data, within the experimental errors of both measurements; however, our values are slightly lower, which could be the result of solvent relaxation during the REMPI process. We attribute the difference between our data and the REMPI photoelectron spectroscopy study of Roy et al. to the good quality of our data at low eKE.

\section{CONCLUSIONS}

We have described the design and characterization of a new recirculating liquid-microjet photoelectron spectrometer for multiphoton ultraviolet photoelectron spectroscopy. It was designed and built for the study of samples that are only available in relatively small quantities, such as chromophores that are not available commercially, proteins, and nanoparticles. We have described the alignment and calibration procedures in detail as well as our approach to characterizing the streaming potential that ensures our measurements are as accurate as possible. We find that the recirculating system improves the quality of the photoelectron spectra at low eKE compared to a liquid nitrogen cold-trap. Interestingly, we find that the streaming potential is zero when we use the recirculating system and we attribute this to the recirculating system grounding the liquid-microjet. 
Multiphoton ultraviolet liquid-microjet photoelectron spectroscopy is a young field and there are still many challenges in the interpretation of liquid-microjet photoelectron spectra. The measured peak positions and widths depend on the reorganization energy of the solute and solvent but are also influenced by inelastic scattering in the liquid-microjet. ${ }^{10,51,53}$ In order to be able to unravel the contribution of electron scattering, it is essential to have high quality photoelectron spectra and to account for, or eliminate, uncertainties arising from streaming potentials. We suspect that comparing high quality multiphoton ultraviolet liquidmicrojet photoelectron spectra and time-resolved photoelectron spectra $^{28-32}$ with analogous measurements in the gas-phase ${ }^{35}$ will provide a new gold standard against which calculations can be benchmarked and can improve our understanding of the role a solvent or protein environment plays in determining electron binding energies and the timescales of relaxation processes of photoexcited molecules.

\section{ACKNOWLEDGMENTS}

This work was supported by the EPSRC (Grant No. EP/L005646/1), the Diamond Light Source (No. STU0157), and the Royal Society and Leverhulme Trust (Grant No. SRF/R1/180079). The authors are grateful to Stephan Figul (Advanced Microfluidic Systems) for technical support and many helpful discussions on running the liquid-microjet.

\section{REFERENCES}

${ }^{1}$ M. N. R. Ashfold, "Photoinitiated quantum molecular dynamics: Concluding remarks," Faraday Discuss. 163, 545-551 (2013).

${ }^{2}$ A. Stolow and J. G. Underwood, "Time-resolved photoelectron spectroscopy," Adv. Chem. Phys. 139, 497-584 (2008).

${ }^{3}$ T. Suzuki, "Time-resolved photoelectron spectroscopy of non-adiabatic electronic dynamics in gas and liquid phases," Int. Rev. Phys. Chem. 31, 265-318 (2012).

${ }^{4}$ R. Spesyvtsev, J. G. Underwood, and H. H. Fielding, "Time-resolved photoelectron spectroscopy for excited state dynamics," in Ultrafast Phenomena in Molecular Sciences: Femtosecond Physics and Chemistry, Springer Series in Chemical Physics Vol. 107, edited by R. de Nalda and L. Banares (Springer, 2014), pp. 99-117.

${ }^{5}$ V. G. Stavros and J. R. R. Verlet, "Gas-phase femtosecond particle spectroscopy: A bottom-up approach to nucleotide dynamics," Annu. Rev. Phys. Chem. 67, 211232 (2016).

${ }^{6}$ H. H. Fielding and G. A. Worth, "Using time-resolved photoelectron spectroscopy to unravel the electronic relaxation dynamics of photoexcited molecules," Chem. Soc. Rev. 47, 309-321 (2018).

${ }^{7}$ M. Bauer, A. Marienfeld, and M. Aeschlimann, "Hot electron lifetimes in metals probed by time-resolved two-photon photoemission," Prog. Surf. Sci. 90, 319-376 (2015).

${ }^{8}$ M. Reutzel, A. Li, and H. Petek, "Coherent two-dimensional multiphoton photoelectron spectroscopy of metal surfaces," Phys. Rev. X 9, 011044 (2019).

${ }^{9}$ M. Faubel, B. Steiner, and J. P. Toennies, "Photoelectron spectroscopy of liquid water, some alcohols, and pure nonane in free micro jets," J. Chem. Phys. 106, 9013 (1997)

${ }^{10} \mathrm{R}$. Seidel, B. Winter, and S. Bradforth, "Valence electronic structure of aqueous solutions: Insights from photoelectron spectroscopy," Annu. Rev. Phys. Chem. 67, 283-305 (2016).

${ }^{11}$ R. Seidel, M. N. Pohl, H. Ali, B. Winter, and E. F. Aziz, "Advances in liquid phase soft-x-ray photoemission spectroscopy: A new experimental setup at BESSY II," Rev. Sci. Instrum. 88, 073107 (2017).
${ }^{12}$ Y. Tang, Y.-i. Suzuki, H. Shen, K. Sekiguchi, N. Kurahashi, K. Nishizawa, P. Zuo, and T. Suzuki, "Time-resolved photoelectron spectroscopy of bulk liquids at ultra-low kinetic energy," Chem. Phys. Lett. 494, 111-116 (2010).

${ }^{13}$ Y. Tang, H. Shen, K. Sekiguchi, N. Kurahashi, T. Mizuno, Y.-I. Suzuki, and T. Suzuki, "Direct measurement of vertical binding energy of a hydrated electron," Phys. Chem. Chem. Phys. 12, 3653 (2010).

${ }^{14}$ Y. I. Yamamoto, S. Karashima, S. Adachi, and T. Suzuki, "Wavelength dependence of UV photoemission from solvated electrons in bulk water, methanol, and ethanol," J. Phys. Chem. A 120, 1153-1159 (2016).

${ }^{15}$ N. Kurahashi, S. Karashima, Y. Tang, T. Horio, B. Abulimiti, Y.-I. Suzuki, Y. Ogi, M. Oura, and T. Suzuki, "Photoelectron spectroscopy of aqueous solutions: Streaming potentials of $\mathrm{NaX}(\mathrm{X}=\mathrm{Cl}, \mathrm{Br}$, and I) solutions and electron binding energies of liquid water and $\mathrm{X}^{-},{ }^{\prime}$ J. Chem. Phys. 140, 174506 (2014).

${ }^{16}$ T. Horio, H. Shen, S. Adachi, and T. Suzuki, "Photoelectron spectra of solvated electrons in bulk water, methanol, and ethanol," Chem. Phys. Lett. 535, 12-16 (2012).

${ }^{17}$ H. Shen, N. Kurahashi, T. Horio, K. Sekiguchi, and T. Suzuki, "Direct measurement of vertical electron binding energies of solvated electrons in methanol and ethanol," Chem. Lett. 39, 668-670 (2010).

${ }^{18}$ S. Karashima, Y.-i. Yamamoto, and T. Suzuki, "Resolving nonadiabatic dynamics of hydrated electrons using ultrafast photoemission anisotropy," Phys. Rev. Lett. 116, 137601 (2016).

${ }^{19}$ Y.-i. Yamamoto, Y. I. Suzuki, G. Tomasello, T. Horio, S. Karashima, R. Mitríc, and T. Suzuki, "Time- and angle-resolved photoemission spectroscopy of hydrated electrons near a liquid water surface," Phys. Rev. Lett. 112, 187603 (2014).

${ }^{20}$ H. Okuyama, Y.-I. Suzuki, S. Karashima, and T. Suzuki, "Charge-transferto-solvent reactions from $\mathrm{I}^{-}$to water, methanol, and ethanol studied by timeresolved photoelectron spectroscopy of liquids," J. Chem. Phys. 145, 074502 (2016).

${ }^{21}$ M. H. Elkins, H. L. Williams, A. T. Shreve, and D. M. Neumark, "Relaxation mechanism of the hydrated electron," Science 342, 1496-1499 (2013).

${ }^{22}$ A. T. Shreve, T. A. Yen, and D. M. Neumark, "Photoelectron spectroscopy of hydrated electrons," Chem. Phys. Lett. 493, 216-219 (2010).

${ }^{23}$ A. T. Shreve, M. H. Elkins, and D. M. Neumark, "Photoelectron spectroscopy of solvated electrons in alcohol and acetonitrile microjets," Chem. Sci. 4, 1633 (2013).

${ }^{24}$ M. H. Elkins, H. L. Williams, and D. M. Neumark, "Dynamics of electron solvation in methanol: Excited state relaxation and generation by charge-transfer-tosolvent," J. Chem. Phys. 142, 234501 (2015).

${ }^{25}$ M. H. Elkins, H. L. Williams, and D. M. Neumark, "Isotope effect on hydrated electron relaxation dynamics studied with time-resolved liquid jet photoelectron spectroscopy," J. Chem. Phys. 144, 184503 (2016).

${ }^{26}$ H. L. Williams, B. A. Erickson, and D. M. Neumark, "Time-resolved photoelectron spectroscopy of adenosine and adenosine monophosphate photodeactivation dynamics in water microjets," J. Chem. Phys. 148, 194303 (2018).

${ }^{27}$ G. Kumar, A. Roy, R. S. Mcmullen, S. Kutagulla, and S. E. Bradforth, "The influence of aqueous solvent on the electronic structure and non-adiabatic dynamics of indole explored by liquid-jet photoelectron spectroscopy," Faraday Discuss. 212, 359-381 (2018)

${ }^{28}$ A. Roy, R. Seidel, G. Kumar, and S. E. Bradforth, "Exploring redox properties of aromatic amino acids in water: Contrasting single photon vs resonant multiphoton ionization in aqueous solutions," J. Phys. Chem. B 122, 3723-3733 (2018).

${ }^{29}$ F. Buchner, A. Lübcke, N. Heine, and T. Schultz, "Time-resolved photoelectron spectroscopy of liquids," Rev. Sci. Instrum. 81, 113107 (2010).

${ }^{30}$ F. Buchner, H.-H. Ritze, J. Lahl, and A. Lübcke, "Time-resolved photoelectron spectroscopy of adenine and adenosine in aqueous solution," Phys. Chem. Chem. Phys. 15, 11402-11408 (2013).

${ }^{31}$ F. Buchner, A. Nakayama, S. Yamazaki, H.-H. Ritze, and A. Lübcke, "Excitedstate relaxation of hydrated thymine and thymidine measured by liquid-jet photoelectron spectroscopy: Experiment and simulation," J. Am. Chem. Soc. 137, 2931-2938 (2015). 
${ }^{32}$ F. Buchner, B. Heggen, H.-H. Ritze, W. Thiel, and A. Lübcke, "Excitedstate dynamics of guanosine in aqueous solution revealed by time-resolved photoelectron spectroscopy: Experiment and theory," Phys. Chem. Chem. Phys. 17, 31978-31987 (2015).

${ }^{33}$ F. Buchner, T. Schultz, and A. Lübcke, "Solvated electrons at the water-air interface: Surface versus bulk signal in low kinetic energy photoelectron spectroscopy," Phys. Chem. Chem. Phys. 14, 5837-5842 (2012).

${ }^{34}$ A. Lübcke, F. Buchner, N. Heine, I. V. Hertel, and T. Schultz, "Time-resolved photoelectron spectroscopy of solvated electrons in aqueous NaI solution," Phys. Chem. Chem. Phys. 12, 14629-14634 (2010).

${ }^{35}$ J. W. Riley, B. Wang, J. L. Woodhouse, M. Assmann, G. A. Worth, and H. H. Fielding, "Unravelling the role of an aqueous environment on the electronic structure and ionization of phenol using photoelectron spectroscopy," J. Phys. Chem. Lett. 9, 678-682 (2018).

${ }^{36}$ B. Winter, "Liquid microjet for photoelectron spectroscopy," Nucl. Instrum. Methods Phys. Res., Sect. A 601, 139-150 (2009).

${ }^{37}$ S. Z. Mikhail and W. R. Kimel, "Densities and viscosities of methanol-water mixtures," J. Chem. Eng. Data 8, 323-328 (1963).

${ }^{38}$ A. Charvat, E. Lugovoj, M. Faubel, and B. Abel, "New design for a time-of-flight mass spectrometer with a liquid beam laser desorption ion source for the analysis of biomolecules," Rev. Sci. Instrum. 75, 1209-1218 (2004).

${ }^{39}$ W. L. Holstein, L. J. Hayes, E. M. C. Robinson, G. S. Laurence, and M. A. Buntine, "Aspects of electrokinetic charging in liquid microjets," J. Phys. Chem. B 103, 3035-3042 (1999).

${ }^{40} \mathrm{P}$. Kruit and F. H. Read, "Magnetic field paralleliser for $2 \pi$ electronspectrometer and electron-image magnifier," J. Phys. E: Sci. Instrum. 16, 313-324 (1983).

${ }^{41}$ A. M. Rijs, E. H. G. Backus, C. A. De Lange, N. P. C. Westwood, and M. H. M. Janssen, "Magnetic bottle' spectrometer as a versatile tool for laser photoelectron spectroscopy," J. Electron Spectrosc. Relat. Phenom. 112, 151-162 (2000).

${ }^{42}$ A. Kothe, J. Metje, M. Wilke, A. Moguilevski, N. Engel, R. Al-Obaidi, C. Richter, R. Golnak, I. Y. Kiyan, and E. F. Aziz, "Time-of-flight electron spectrometer for a broad range of kinetic energies," Rev. Sci. Instrum. 84, 023106 (2013).
${ }^{43}$ G. K. Jarvis, M. Evans, C. Y. Ng, and K. Mitsuke, "Rotational-resolved pulsed field ionization photoelectron study of NO in the energy range of 9.24-16.80 eV," J. Chem. Phys. 111, 3058 (1999).

${ }^{44}$ J. O. Bockris, M. A. V. Devanathan, and K. Muller, "On the structure of charged interfaces,” Proc. R. Soc. London, Ser. A 274, 55-79 (1963).

${ }^{45} \mathrm{~B}$. Winter and M. Faubel, "Photoemission from liquid aqueous solutions," Chem. Rev. 106, 1176-1211 (2006).

${ }^{46}$ N. Preissler, F. Buchner, T. Schultz, and A. Lübcke, "Electrokinetic charging and evidence for charge evaporation in liquid microjets of aqueous salt solution," J. Phys. Chem. B 117, 2422-2428 (2013).

${ }^{47} \mathrm{M}$. Faubel and B. Steiner, "Strong bipolar electrokinetic charging of thin liquid jets emerging from $10 \mu \mathrm{m}$ PtIr nozzles," Ber. Bunsengesellschaft Phys. Chem. 96, 1167-1172 (1992).

${ }^{48}$ M. Faubel, B. Steiner, and J. Toennies, "Measurement of HeI photoelectron spectra of liquid water, formamide and ethylene glycol in fast-flowing microjets," J. Electron Spectrosc. Relat. Phenom. 95, 159-169 (1998).

${ }^{49}$ R. Weber, B. Winter, P. M. Schmidt, W. Widdra, I. V. Hertel, M. Dittmar, and M. Faubel, "Photoemission from aqueous alkali-metal-iodide salt solutions using EUV synchrotron radiation," J. Phys. Chem. B 108, 4729-4736 (2004).

${ }^{50}$ D. Ghosh, A. Roy, R. Seidel, B. Winter, S. Bradforth, and A. I. Krylov, "Firstprinciple protocol for calculating ionization energies and redox potentials of solvated molecules and ions: Theory and application to aqueous phenol and phenolate," J. Phys. Chem. B 116, 7269-7280 (2012).

${ }^{51}$ P. R. Tentscher, R. Seidel, B. Winter, J. J. Guerard, and J. S. Arey, "Exploring the aqueous vertical ionization of organic molecules by molecular simulation and liquid microjet photoelectron spectroscopy," J. Phys. Chem. B 119, 238-256 (2015).

${ }^{52}$ M. A. Brown, Z. Abbas, A. Kleibert, R. G. Green, A. Goel, S. May, and T. M. Squires, "Determination of surface potential and electrical double-layer structure at the aqueous electrolyte-nanoparticle interface," Phys. Rev. X 6, 011007 (2016).

${ }^{53}$ D. Luckhaus, Y.-i. Yamamoto, T. Suzuki, and R. Signorell, "Genuine binding energy of the hydrated electron," Sci. Adv. 3, e1603224 (2017). 